\title{
Atrial fibrillation with a giant left atrial appendage can be successfully treated with pulmonary vein antrum isolation
}

\author{
I. E. Hof • T. X. Wildbergh • V. J. van Driel • \\ F. H. Wittkampf • M. J. Cramer • M. Meine • \\ R. N. Hauer • P. Loh
}

Published online: 21 May 2011

(C) The Author(s) 2011. This article is published with open access at Springerlink.com

\section{Introduction}

A congenital giant left atrial appendage (LAA) is a rare condition only described in case reports [1]. Supraventricular tachycardias, including atrial fibrillation (AF), and systemic embolisation are the most common clinical findings $[1,2]$. Almost all reported cases are treated by resection of the giant LAA [1]. A few cases are treated conservatively $[3,4]$. Catheter ablation has not been reported as a treatment option. For the first time, we present a patient with paroxysmal AF and a congenital giant LAA who was successfully treated by percutaneous pulmonary vein antrum isolation (PVAI).

\section{Case}

A 42-year-old man with drug-refractory AF was referred to our cardiology outpatient clinic to discuss treatment options. Echocardiography and magnetic resonance imaging (MRI) had been performed in the referring hospital which had revealed a giant LAA. No other abnormalities were reported.

The main complaint of the patient was recurrent, nocturnal episodes of AF once a week. His medical history

\footnotetext{
I. E. Hof • V. J. van Driel • F. H. Wittkampf • M. J. Cramer •

M. Meine $\cdot$ R. N. Hauer $\cdot$ P. Loh $(\bowtie)$

Department of Cardiology, E03.511, University Medical Center,

PO Box 85500, 3508 Utrecht, the Netherlands

e-mail: p.loh@umcutrecht.nl
}

T. X. Wildbergh

Department of Cardiology, Meander Medical Center,

Amersfoort, the Netherlands was unremarkable. Physical examination revealed no abnormalities and electrocardiography showed sinus rhythm without signs of left atrial enlargement. The chest radiograph showed a prominent left upper cardiac border but with a normal cardiothoracic ratio. Sotalol, and later amiodarone, flecainide and disopyramide were tried but were ineffective or resulted in unpleasant side effects.

Since medical therapy was no option, alternative treatments were discussed. The role of the giant LAA in the pathophysiology of AF is unclear. Therefore, we proposed to perform PVAI by radiofrequency catheter ablation leaving the giant LAA in situ. The patient gave his consent.

In preparation for PVAI, transoesophageal echocardiography was performed which showed a giant LAA with good flow velocity of $>40 \mathrm{~cm} / \mathrm{s}$ (Fig. 1). No thrombi were seen. MRI was repeated and revealed the giant LAA with a length of $7 \mathrm{~cm}$ in the coronal view (Figs. 2 and 3).

PVAI was performed by encircling right and left pulmonary vein antrums in pairs using an irrigated $4 \mathrm{~mm}$ electrode with a maximum electrode temperature of $43^{\circ} \mathrm{C}$ and a power of 30-40 W. The procedure lasted $240 \mathrm{~min}$ and the endpoint was successful electrical isolation of all pulmonary veins. Total fluoroscopy dose was $104 \mathrm{mGy}$ and total fluoroscopy time was $27 \mathrm{~min}$. The course of the procedure was uneventful.

After PVAI the patient was seen at the outpatient clinic. Antiarrhythmic medication was discontinued after 3 months. Six months after ablation the patient experienced a single episode of AF, treated with electrical cardioversion. With the exception of this event no recurrences of AF were seen, confirmed by four 48-hour Holter recordings (5, 7, 12, and 16 months following PVAI). Seventeen months after PVAI the patient is still in sinus rhythm and asymptomatic. He will continue to take oral anticoagulation. 
Fig. 1 Transoesophageal echocardiogram showing the giant left atrial appendage. $\mathrm{LA}=$ left atrium, LAA = left atrial appendage, $L V=$ left ventricle, $\mathrm{MV}=$ mitral valve

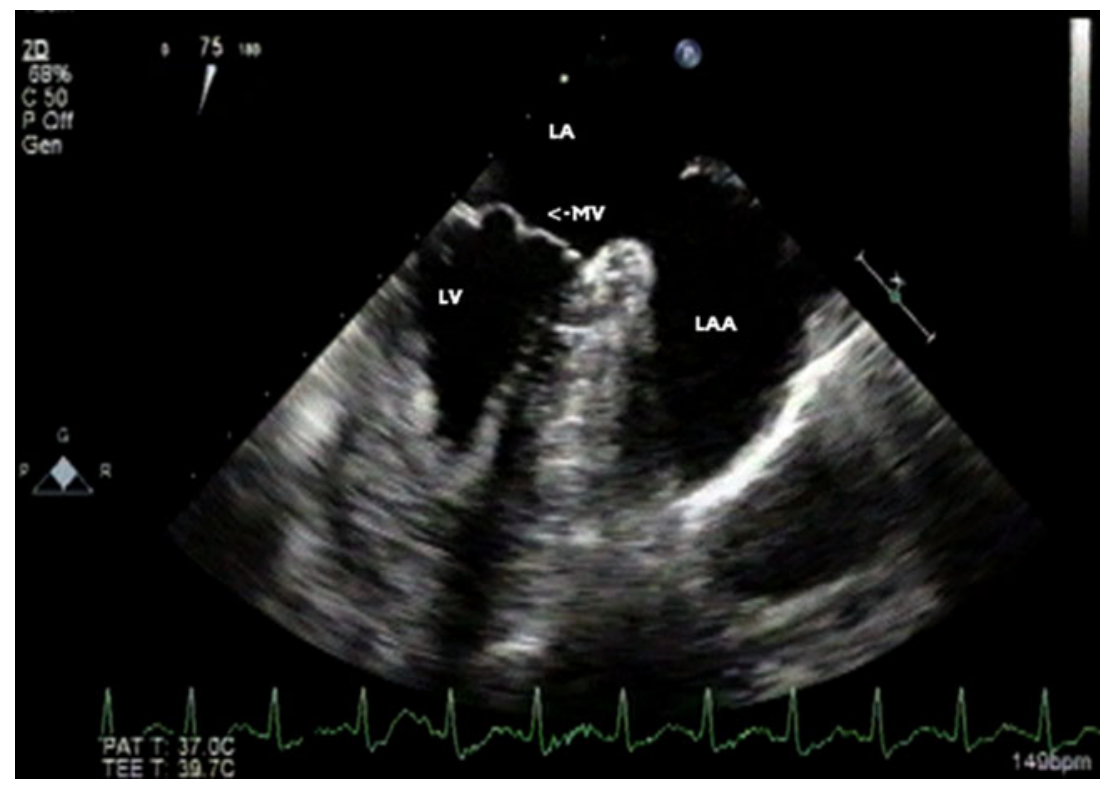

\section{Discussion}

A giant LAA is a rare condition that can be acquired or congenital when it presents as an isolated anomaly, as was the case in our patient $[5,6]$. The patient has a monozygotic twin brother who also underwent MRI; however, no abnormalities were seen.

Approximately 80 case reports were identified by Chowdhury et al. that describe an aneurysm of the LAA [1]. Almost all known cases are treated by resection of the LAA and it is advised in the literature to perform this treatment even in asymptomatic patients to prevent $\mathrm{AF}$ and thromboembolic

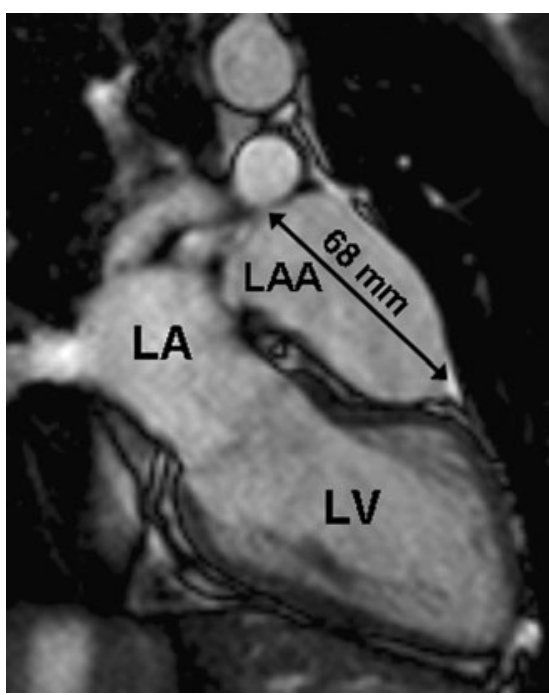

Fig. 2 Magnetic resonance imaging of the heart in oblique coronal view demonstrating the giant left atrial appendage with a length of $68 \mathrm{~mm} . \mathrm{LA}=$ left atrium, $\mathrm{LAA}=$ left atrial appendage, $\mathrm{LV}=$ left ventricle events [1, 2, 7, 8]. However, a role of the giant LAA as a substrate of AF has never been demonstrated and no large trials have been performed to test the efficacy of resection of a giant LAA in patients with AF. Mathur et al. performed an electrophysiological study in a patient with a giant LAA presenting with AF and found arrhythmic foci in both atria quickly degenerating into AF [9]. They decided to perform a Cox-Maze III procedure in addition to LAA resection since they hypothesised that the trigger of AF may not be located in the giant LAA itself. One year post-operatively this patient was still in sinus rhythm. Therefore, on the grounds of the present literature, the role of the giant LAA in the aetiology of AF is unknown.

In 1998 Haissaguerre et al. identified pulmonary vein triggers as a principal mechanism of AF initiation and perpetuation [10]. Since then, electrical isolation of the

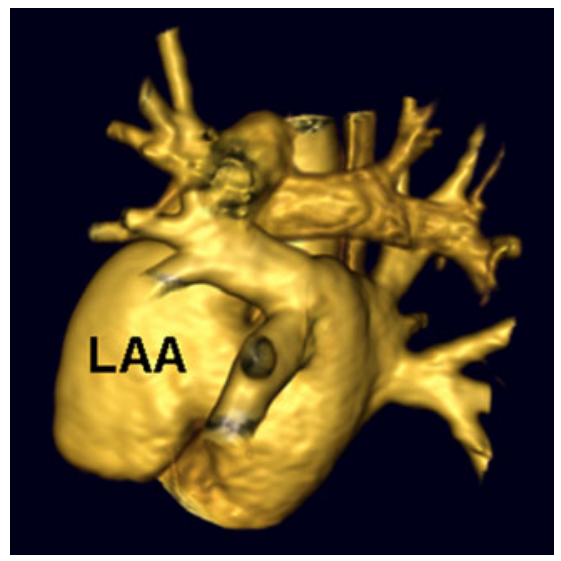

Fig. 3 Three-dimensional reconstruction with magnetic resonance imaging showing the left atrium with the giant left atrial appendage from left posterior. LAA $=$ left atrial appendage 
pulmonary veins by catheter ablation has become the treatment of choice in patients with AF [11]. Since our patient had typically paroxysmal AF with normal left atrial size we hypothesised that the trigger for AF may be located in the pulmonary veins and not in the LAA. Therefore, we decided to perform PVAI without resection of the LAA. With the exception of one episode, this patient had no recurrences of AF while off antiarrhythmic drugs 17 months after the procedure, providing evidence for a pulmonary vein trigger.

\section{Conclusion}

This case report describes a patient with a congenital giant LAA presenting with typically vagally induced paroxysmal AF. We have shown that this patient can be successfully treated with PVAI without resection of the LAA.

Disclosures F.H. Wittkampf is a consultant for St. Jude Medical.

Open Access This article is distributed under the terms of the Creative Commons Attribution Noncommercial License which permits any noncommercial use, distribution, and reproduction in any medium, provided the original author(s) and source are credited.

\section{References}

1. Chowdhury UK, Seth S, Govindappa R, et al. Congenital left atrial appendage aneurysm: a case report and brief review of literature. Heart Lung Circ. 2009;18:412-6.

2. Zhao J, Ge Y, Yan H, et al. Treatment of congenital aneurysms of the left atrium and left atrial appendage. Tex Heart Inst J. 1999;26:136-9.

3. Thomas E, Salmon AP, Vettukattil JJ. Intrapericardial giant left atrial appendage. Cardiol Young. 2004;14:338-40.

4. Lekkerkerker JC, Jaarsma W, Cramer MJ. Congenital giant aneurysm of the left atrial appendage. Heart. 2005;91:e21.

5. Pome G, Pelenghi S, Grassi M, et al. Congenital intrapericardial aneurysm of the left atrial appendage. Ann Thorac Surg. 2000;69:1569-71.

6. Bramlet DA, Edwards JE. Congenital aneurysm of left atrial appendage. Br Heart J. 1981;45:97-100.

7. Soleimani A, Sattarzadeh R. Left atrial appendage aneurysm: a rare cause of paroxysmal supraventricular tachycardia. Heart Lung Circ. 2008;17:246-7.

8. Kiaii B, Doll N, Kuehl M, et al. Minimal invasive endoscopic resection of a giant left atrial appendage aneurysm. Ann Thorac Surg. 2004;77:1437-8.

9. Mathur A, Zehr KJ, Sinak LJ, et al. Left atrial appendage aneurysm. Ann Thorac Surg. 2005;79:1392-3.

10. Haissaguerre M, Jais P, Shah DC, et al. Spontaneous initiation of atrial fibrillation by ectopic beats originating in the pulmonary veins. N Engl J Med. 1998;339:659-66.

11. Camm AJ, Kirchhof P, Lip GY, et al. Guidelines for the management of atrial fibrillation: the Task Force for the Management of Atrial Fibrillation of the European Society of Cardiology (ESC). Europace. 2010;12:1360-420. 